\title{
Impact of Mining on Nutritional Status of Women of Reproductive Age Residing in Mining Affected Talukas of Ballari District, India
}

\author{
K. Y. Ullikashi*' and U.N. Kulkarni \\ Department of Food Science and Nutrition, College of Community Science, \\ UAS, Dharwad-580005, India \\ *Corresponding author
}

\section{A B S T R A C T}

\section{Keywords}

Mining area, Non mining area,

Anthropometry,

Anemia and clinical symptoms

Article Info

Accepted:

12 December 2020

Available Online:

10 January 2021
A study on impact of mining on nutritional status of women of reproductive age was carried out in two mining affected talukas namely, Hospet and Sandur of Ballari district. A total of 140 women were selected from mining and non mining area. Nutritional status of women was assessed using anthropometry, dietary, clinical and biochemical analysis. Majority of the women residing in mining were overweight (24.2\%) and obese (18.5\%), where 42.8 per cent of women residing in non mining were undernourished. The intake of food and nutrients were significantly higher in women residing in mining area compared to non mining area. Women residing in both mining and non mining area were categorized as mild anemic (10.06 g/dl), but prevalence was higher $(55.7 \%)$ in women residing non mining area. Prevalence of clinical symptoms clinical symptoms such as pallor skin, lassitude, lack of interest, irritation, anorexia and glossitis were higher $(81.4,82.8,90.0,90.0,45.7$ and 82.8 per cent respectively) in women residing in non mining area compared to mining area $(58.5,58.5,585,57.5$ and 52.8 per cent respectively).

\section{Introduction}

Mining is a major economic activity in many developing countries like India, China, South Africa and Brazil. Whether it is of small or large scale are inherently destructive to the environment. During different stages of mining, it adversely affects the natural environment and cultural heritage at both macro and micro level (Patra et al., 2015). In micro level due to gender equality domain in the family and community, women for the most are negatively affected well being. Though women directly not involved in mining activities but expose to harsh environment during water and fire wood collection leads to enormous health problems (Yeboah, 2008). Despite people suffering from ill health, mental and physical deformities and constant exposure to toxics 
waste and chronic diseases due to mining, there is a tragic gap in the availability of scientific study and data on the health hazards of mining in India and more so on the women in mining affected communities (Anonymous, 2013). Hence the study was carried out to assess the impact of mining on nutritional status of women in reproductive age residing in mining affected talukas of Bellari district

\section{Materials and Methods}

Study carried out in two mining affected talukas namely Hospet and Sandur of Bellari district. In each taluka two villages were selected in mining and non mining area. Total 140 women of reproductive age $(15-45$ years) were selected for study. The height, weight, waist and hip circumference of women were measured. The basal metabolic index (BMI) and waist to hip ratio (WHR) were calculated and further classified based on grades of malnutrition (Jelliffe, 1966). Diet survey was conducted by using 24 hour recall method. The percent adequacy of nutrients was computed using recommended dietary allowance (RDA) given by ICMR (Anon., 2020). Clinical survey was done using rapid clinical survey given by National Institute of Nutrition (NIN ) (Anon., 2003). Hemoglobin status of women was assessed by cyanomethemoglobin method (Verely, 1976) using Drabkins reagent. The data was analysed using SPSS 16.00 version.

\section{Results and Discussion}

Mining industry in India is a major activity which contribute significantly to the economy of India, but has remained a major environmental concern. This results in direct and indirect impact on women by reduction in quality of life due to denial of access to food security, natural resources and livelihoods. The woman residing both in mining (45.7\%) and non mining $(50.0 \%)$ were educated up to high School, where as 4.2 per cent women had completed degree in mining area. Majority of women in mining women were belonged to schedule tribe (40.1), where as 41.4 per cent of women residing in non mining area belong to backward class. Majority of both women were non vegetarians $(82.8 \%)$ and mean age at marriage was below 18 years (17.15 and 17.25 years respectively).

Table 2. Indicates classification of selected women based on BMI and WHR residing in mining and non mining area. The mean weight and waist circumference of women residing in mining area were $53.17 \mathrm{Kgs}$ and $85.7 \mathrm{~cm}$ respectively which were significantly higher compared to non mining area (45.3 Kgs and $78.3 \mathrm{cms}$ respectively). The height and hip circumference values were did not differ significantly in both mining (148.5 and $96.5 \mathrm{~cm}$ respectively) and non mining areas (151.1 and $93.4 \mathrm{~cm}$ respectively) (Fig.1). Prevalence of obesity and overweight was high in women of mining area (24.2 and 18.5 $\%$ respectively) compared to non mining area (18.5 and $1.42 \%$ respectively), whereas prevalence of under nutrition was high (42.8 $\%)$ in women residing in non mining area compared to women residing in mining area $(12.8 \%)$. Similarly based on waist to hip ratio, 74.1 per cent women of mining area were categorized as at high risk where as 60.0 per cent women of non mining area were categorized as at moderate risk. Similar results were also found in studies of Belashsen et al., (2003); Rao et al., (2010) and Mittal (2003).

Mean intake of food of selected women in mining and non mining area was shown in table 3 and Figure 2. Cereal and millets in both mining (304.7 g / ACU / day) and non mining women (294.3 g / ACU/ day) were above the recommended dietary allowances (RDA), where as intake of fat and sugar in women of mining area (22.5 and $21.5 \mathrm{~g} /$ 
ACU / day respectively) was significantly higher compared to non mining (18.0 and $17.5 \mathrm{~g} / \mathrm{ACU} / \mathrm{day}$ respectively) and RDA. Mean intake of pulses, meat, egg, fish and other vegetables were high in women of mining area compared to non mining but were less than RDA. Consumption of milk and milk products, roots and tubers and fruits was slightly higher in women residing in non mining area (73.95, 84.8 and 25.35 g / ACU / day respectively) compared to mining area (69.0, 65.6 and $23.3 \mathrm{~g} / \mathrm{ACU} /$ day respectively) but were less than recommended dietary allowance. Similarly, except energy, crude fiber, copper and zinc all the nutrients intake was significantly higher in women residing mining area compared to non mining area but were less than recommended dietary allowances (Table 4, 5 and 6). Results of the study confirm with the results of Mittal (2003) ; Rao et al., (2010) and Mamata and Devi (2014).

Table 7 shows the frequency and consumption of food rich in blood-forming nutrients in mining and non mining areas. The result reveal that mean intake of carrot, pumpkin, yellow coloured fruits and vegetables, guva, amla, pineapple, citrus fruits and fermented food were high in women residing in mining area $(2.80,2.48,3.01,1.68$, $0.38,0.40,1.44,2.80,3.45,4.00$ and 3.54 respectively) compared to women residing in non mining $(2.35,1.74,2.91,1.18,0.28,0.21$, $0.85,2.31,2.70,3.04$ and 2.52 respectively).

Table.1 Demographic profile of selected women in mining and non mining areas

\begin{tabular}{|c|c|c|c|c|c|}
\hline \multirow{3}{*}{$\begin{array}{l}\text { Sl } \\
\text { no }\end{array}$} & & & & & \\
\hline & \multirow{2}{*}{ Details } & \multicolumn{2}{|c|}{ Mining area } & \multicolumn{2}{|c|}{ Non mining area } \\
\hline & & $\mathbf{n}$ & $\%$ & $\mathbf{n}$ & $\%$ \\
\hline \multirow[t]{7}{*}{1} & \multicolumn{5}{|l|}{ Education } \\
\hline & Illiterate & 13 & 18.5 & 12 & 17.1 \\
\hline & Primary & 15 & 21.4 & 16 & 22.8 \\
\hline & High school & 32 & 45.7 & 35 & 50.0 \\
\hline & Intermediate & 7 & 10.0 & 7 & 10.0 \\
\hline & Degree & 3 & 4.2 & - & - \\
\hline & Post graduate & - & - & - & - \\
\hline \multirow[t]{5}{*}{2} & \multicolumn{5}{|l|}{ Caste } \\
\hline & Upper caste & 8 & 11.4 & 19 & 27.1 \\
\hline & Backward caste & 16 & 22.8 & 29 & 41.4 \\
\hline & $\mathrm{SC}$ & 18 & 25.7 & 17 & 24.2 \\
\hline & ST & 28 & 40.0 & 5 & 7.14 \\
\hline \multirow[t]{3}{*}{3} & \multicolumn{5}{|l|}{ Type of family } \\
\hline & Nuclear & 40 & 57.1 & 39 & 55.7 \\
\hline & Joint & 30 & 42.8 & 31 & 44.2 \\
\hline \multirow[t]{4}{*}{4} & \multicolumn{5}{|l|}{ Food habits } \\
\hline & Vegetarian & 8 & 11.4 & 12 & 17.1 \\
\hline & Ovo- vegetarian & 4 & 5.71 & - & - \\
\hline & Non vegetarian & 58 & 82.8 & 58 & 82.8 \\
\hline 5 & $\begin{array}{l}\text { Mean age at } \\
\text { marriage }\end{array}$ & \multicolumn{2}{|c|}{$\begin{array}{c}17.25 \\
(14-25)\end{array}$} & \multicolumn{2}{|c|}{$\begin{array}{c}17.15 \\
(14-21)\end{array}$} \\
\hline
\end{tabular}


Table.2 Classification of selected women based on BMI and WHR in mining and non mining areas

\begin{tabular}{|c|c|c|c|c|c|}
\hline \multirow{3}{*}{ SI no } & \multirow{3}{*}{ Details } & \multicolumn{4}{|c|}{$\mathrm{N}=140$} \\
\hline & & \multicolumn{2}{|c|}{ Mining area } & \multicolumn{2}{|c|}{ Non mining area } \\
\hline & & $\mathbf{n}$ & $\%$ & $\mathbf{n}$ & $\%$ \\
\hline \multirow[t]{5}{*}{1} & \multicolumn{5}{|c|}{ Body mass index (BMI) } \\
\hline & Under weight $(<18.5)$ & 9 & 12.8 & 30 & 42.8 \\
\hline & $\begin{array}{l}\text { Normal } \\
(18.5-24.5)\end{array}$ & 31 & 44.2 & 33 & 47.14 \\
\hline & $\begin{array}{l}\text { Over weight } \\
\text { (Grade-1 obesity) } \\
(25.0-30.0)\end{array}$ & 17 & 24.2 & 8 & 8.5 \\
\hline & $\begin{array}{l}\text { Obese } \\
\text { (Grade- } 2 \text { obesity) } \\
(>30.0-40.0)\end{array}$ & 13 & 18.5 & 1 & 1.42 \\
\hline \multicolumn{6}{|c|}{ t- values } \\
\hline \multicolumn{6}{|c|}{ Mining and non mining } \\
\hline \multicolumn{6}{|c|}{$6.665 * *$} \\
\hline \multirow[t]{4}{*}{2} & \multicolumn{5}{|c|}{ Waist to hip ratio (WHR) } \\
\hline & $\begin{array}{l}\text { Low risk } \\
(0.80 \text { or below })\end{array}$ & 8 & 11.4 & 12 & 17.1 \\
\hline & $\begin{array}{l}\text { Moderate risk } \\
(0.81-0.85)\end{array}$ & 12 & 17.1 & 42 & 60.0 \\
\hline & $\begin{array}{l}\text { High risk } \\
(>0.85)\end{array}$ & 50 & 71.4 & 16 & 22.8 \\
\hline \multicolumn{6}{|c|}{ t- values } \\
\hline \multicolumn{6}{|c|}{ Mining and non mining } \\
\hline \multicolumn{6}{|c|}{$5.426 * *$} \\
\hline
\end{tabular}

** - Significant at 0.01 level

Table.3 Mean food intake of selected women in mining and non mining areas

\begin{tabular}{|c|c|c|c|c|c|c|}
\hline \multirow{2}{*}{$\begin{array}{c}\text { Sl } \\
\text { No }\end{array}$} & \multirow{2}{*}{$\begin{array}{c}\text { Food } \\
(\text { g/ACU/day })\end{array}$} & \multirow[t]{2}{*}{ RDA* } & \multicolumn{2}{|c|}{ Mining area } & \multicolumn{2}{|c|}{ Non mining area } \\
\hline & & & Mean & $\%$ adequacy & Mean & $\%$ adequacy \\
\hline 1 & Cereals and millets & 270 & 304.7 & 112.8 & 294.2 & 108.9 \\
\hline 2 & Pulses & 60 & 40.8 & 68.0 & 29.25 & 48.75 \\
\hline 3 & Meat, egg \&fish & 50 & 19.5 & 39.0 & 12.25 & 24.50 \\
\hline 4 & Milk \& Milk products & 300 & 69.0 & 23.0 & 73.95 & 24.65 \\
\hline 6 & Roots and tubers & 200 & 65.6 & 32.8 & 84.85 & 42.4 \\
\hline 7 & Green leafy vegetables & 100 & 35.1 & 35.1 & 34.3 & 34.3 \\
\hline 8 & Other vegetables & 200 & 99.9 & 49.95 & 95.6 & 47.8 \\
\hline 9 & Fruits & 100 & 23.3 & 23.0 & 25.35 & 25.35 \\
\hline 10 & Sugar & 20 & 22.5 & 112.5 & 18.0 & 90.0 \\
\hline 11 & Oils and fat & 20 & 21 & 105.0 & 17.5 & 87.5 \\
\hline 12 & Condiments and spices & & 9.95 & & 10.75 & \\
\hline 13 & Others (Bread, biscuit, papad, pickle) & & 19.25 & & 4.5 & \\
\hline
\end{tabular}

- $\quad$ Anon (2010) 
Table.4 Mean intake of major nutrient of selected women in mining and non mining areas

\begin{tabular}{|l|c|c|c|c|c|c|}
\hline \multicolumn{1}{c|}{$\begin{array}{c}\text { Major } \\
\text { Nutrients } \\
\text { (g/day) }\end{array}$} & $\begin{array}{c}\text { Mining } \\
\text { area }\end{array}$ & $\begin{array}{c}\text { Non mining } \\
\text { area }\end{array}$ & RDA* & \multicolumn{3}{c|}{ t-values } \\
\cline { 5 - 7 } & & & & $\begin{array}{c}\text { Mining } \\
\text { vs non } \\
\text { mining }\end{array}$ & Mining & $\begin{array}{c}\text { Non } \\
\text { mining }\end{array}$ \\
\hline Energy (Kcal) & 1864 & 1785 & 1660 & $1.532^{\text {NS }}$ & $7.42^{* *}$ & $8.24^{* * *}$ \\
\hline Protein & 35.8 & 31.0 & 45.7 & $5.456^{* *}$ & $27.43^{* *}$ & $27.41^{* *}$ \\
\hline Fat & 27.3 & 20.8 & 20 & $7.594^{* *}$ & $10.12^{* *}$ & $0.25^{\text {NS }}$ \\
\hline Crude fiber & 22.2 & 22.3 & 25 & $0.243^{\text {NS }}$ & $6.70^{* *}$ & $6.28^{* *}$ \\
\hline
\end{tabular}

*Anon (2020)

** - Significant at 0.01 level, NS-Non significant

Table.5 Mean intake of minerals of selected women in mining and non mining areas

$(\mathrm{N}=140)$

\begin{tabular}{|l|c|c|c|c|c|c|}
\hline \multicolumn{1}{r}{$\begin{array}{c}\text { Minerals } \\
\text { (mg/day) }\end{array}$} & Mining area & $\begin{array}{c}\text { Non mining } \\
\text { area }\end{array}$ & RDA* & \multicolumn{3}{c|}{ t-values } \\
\cline { 5 - 7 } & & & & $\begin{array}{c}\text { Mining } \\
\text { vs non } \\
\text { mining }\end{array}$ & Mining & $\begin{array}{c}\text { Non } \\
\text { mining }\end{array}$ \\
\hline Calcium & 116 & 149 & 1000 & $4.262^{* *}$ & $19.40^{* *}$ & $13.69^{* *}$ \\
\hline Sodium & 2263 & 1874 & 2000 & $6.038^{* *}$ & $6.99^{* *}$ & $-2.40^{*}$ \\
\hline Potassium & 1723 & 1414 & 3500 & $6.227^{* *}$ & $45.16^{* *}$ & $68.66^{* *}$ \\
\hline Iron & 18.08 & 15.4 & 19 & $4.431^{* *}$ & $1.96^{\text {NS }}$ & $9.84^{* *}$ \\
\hline Copper & 1.07 & 1.04 & 2.0 & $0.883^{\text {NS }}$ & $39.16^{* *}$ & $57.28^{* *}$ \\
\hline Zinc & 7.76 & 7.72 & 17 & $0.199^{\text {NS }}$ & $68.24^{* *}$ & $67.13^{* *}$ \\
\hline
\end{tabular}

*Anon (2020)

** - Significant at 0.01 level, NS-Non significant

Table.6 Mean intake of vitamins of selected women in mining and non mining areas

$(\mathrm{N}=140)$

\begin{tabular}{|l|c|c|c|c|c|c|}
\hline \multicolumn{1}{c|}{$\begin{array}{c}\text { Major } \\
\text { Nutrients } \\
\text { (g/day) }\end{array}$} & $\begin{array}{c}\text { Mining } \\
\text { area }\end{array}$ & $\begin{array}{c}\text { Non mining } \\
\text { area }\end{array}$ & \multirow{2}{*}{ RDA* } & \multicolumn{3}{|c|}{ t-values } \\
\cline { 5 - 7 } & & & & $\begin{array}{c}\text { Mining } \\
\text { vs non } \\
\text { mining }\end{array}$ & Mining & $\begin{array}{c}\text { Non } \\
\text { mining }\end{array}$ \\
\hline Thiamin & 0.96 & 0.83 & 1.4 & $5.483^{* *}$ & $22.76^{* *}$ & $33.04^{* *}$ \\
\hline Riboflavin & 0.72 & 0.65 & 2.0 & $2.994^{* *}$ & $6.29^{* *}$ & $9.75^{* *}$ \\
\hline Pyridoxine & 0.880 .95 & 0.86 & 1.9 & $2.484^{*}$ & $8.04^{* *}$ & $9.31^{* *}$ \\
\hline Vitamin A & 275 & 188 & 840 & $8.873^{* *}$ & $7.00^{* *}$ & $10.04^{* *}$ \\
\hline Carotene (ug) & 823 & 771 & 6720 & $2.114^{*}$ & $34.85^{* *}$ & $32.18^{* *}$ \\
\hline Choline & 89 & 83 & & $0.912^{\text {NS }}$ & & \\
\hline Folic acid (ug) & 36.6 & 29.6 & 100 & $8.677^{* *}$ & $12.66^{* *}$ & $13.83^{* *}$ \\
\hline Niacin & 7.82 & 7.89 & 14 & $0.140^{\text {NS }}$ & $8.175^{* *}$ & $5.972^{* *}$ \\
\hline Vitamin B12 & 0.92 & 0.80 & 2.5 & $5.793^{* *}$ & $8.495^{* *}$ & $7.33^{* *}$ \\
\hline Vitamin C & 34.2 & 23.5 & 80 & $10.059^{* *}$ & $15.64^{* *}$ & $18.53^{* *}$ \\
\hline
\end{tabular}

*Anon (2020)

** - Significant at 0.01 level, NS-Non significant 
Table.7 Distribution of women based on frequency of consumption of foods rich in blood forming nutrients in mining and non mining areas

\begin{tabular}{|c|c|c|c|c|c|c|c|c|c|c|c|c|c|c|c|}
\hline \multirow[t]{2}{*}{ SI no } & \multirow[t]{2}{*}{ Items } & \multicolumn{6}{|c|}{ Mining area } & \multirow[t]{2}{*}{ Mean } & \multicolumn{6}{|c|}{ Non mining area } & \multirow[t]{2}{*}{ Mean } \\
\hline & & D & $\mathbf{T}$ & $\mathbf{W}$ & $\mathbf{F}$ & $\mathbf{O}$ & $\mathbf{N}$ & & D & $\mathbf{T}$ & $\mathbf{W}$ & $\mathbf{F}$ & $\mathbf{O}$ & $\mathbf{N}$ & \\
\hline 1 & $\begin{array}{l}\text { Green leafy } \\
\text { vegetables }\end{array}$ & 8 & 2 & 60 & - & - & - & 2.40 & - & 3 & 66 & 1 & - & - & 3.04 \\
\hline 2 & $\begin{array}{l}\text { Other } \\
\text { vegetables }\end{array}$ & 2 & 64 & 3 & - & - & - & 3.92 & 1 & 63 & 5 & 1 & - & - & 3.91 \\
\hline 3 & Sweets & - & 3 & 36 & 31 & - & - & 1.61 & - & 2 & 26 & 35 & 7 & - & 2.32 \\
\hline 4 & Carrot & - & 3 & 59 & 7 & - & 1 & 2.80 & - & - & 32 & 31 & 7 & - & 2.35 \\
\hline 5 & Pumpkin & - & - & 28 & 40 & 10 & 9 & 2.48 & - & - & 5 & 44 & 19 & 1 & 1.74 \\
\hline 6 & $\begin{array}{l}\text { Yellow coloured } \\
\text { fruits/vegetables }\end{array}$ & - & 17 & 47 & 1 & - & 5 & 3.01 & - & 5 & 54 & 11 & - & - & 2.91 \\
\hline 7 & $\begin{array}{l}\text { Germinated } \\
\text { grains }\end{array}$ & - & 22 & 28 & 12 & - & - & 2.80 & - & 20 & 22 & 28 & - & - & 2.31 \\
\hline 8 & Guava & - & - & 2 & 51 & 10 & 7 & 1.68 & - & - & - & 13 & 57 & - & 1.18 \\
\hline 9 & Amla & - & 1 & 3 & - & 17 & 49 & 0.38 & - & - & - & 5 & 10 & 45 & 0.28 \\
\hline 10 & $\begin{array}{l}\text { Fermented } \\
\text { products }\end{array}$ & 11 & 26 & 30 & 2 & 1 & - & 3.62 & - & 8 & 20 & 35 & 15 & - & 2.52 \\
\hline 11 & Pineapple & - & - & - & 2 & 24 & 54 & 0.40 & - & - & - & 2 & 11 & 57 & 0.21 \\
\hline 12 & Citrus fruits & - & 1 & 25 & 9 & 4 & 31 & 1.44 & - & 1 & 10 & 10 & 6 & 33 & 0.85 \\
\hline 13 & $\begin{array}{l}\text { Milk and milk } \\
\text { products }\end{array}$ & 10 & 8 & 52 & - & - & - & 3.28 & 6 & 8 & 56 & - & - & - & 3.28 \\
\hline 14 & Animal foods & - & 56 & 6 & - & - & 8 & 3.45 & - & 21 & 35 & - & - & 14 & 2.70 \\
\hline 15 & Egg & - & 60 & 2 & - & - & 8 & 3.54 & - & 45 & 11 & - & - & 7 & 3.04 \\
\hline 16 & Ground nut & - & 70 & - & - & - & - & 4.00 & 6 & 56 & 8 & - & - & - & 3.97 \\
\hline
\end{tabular}

D - Daily; TW - Twice a week; W - Weekly; F - Fortnightly O - Occasionally N-Never

Score system : 5 - Daily; 4 - Twice a week; 3 - Weekly; 2 - Fortnightly, 1 - Occasionally, Never-0

Table.8 Distribution of women based on hemoglobin level in mining and non mining areas

\begin{tabular}{|c|c|c|c|c|}
\hline \multirow[b]{2}{*}{$\begin{array}{c}(\mathrm{N}=140) \text { Haemoglobin } \\
\text { level } \\
(\mathrm{g} / \mathrm{dl})\end{array}$} & \multicolumn{2}{|c|}{ Mining area } & \multicolumn{2}{|c|}{ Non mining area } \\
\hline & $\mathbf{n}$ & $\%$ & $\mathbf{n}$ & $\%$ \\
\hline Normal (>12.0) & 29 & 41.4 & 7 & 10 \\
\hline $\begin{array}{l}\text { Mild anemia (10.5 - } \\
11.99)\end{array}$ & 31 & 44.2 & 39 & 55.7 \\
\hline Moderate (8.0 - 10.49) & 9 & 12.8 & 24 & 34.2 \\
\hline Severe $(<8.0)$ & 1 & 1.42 & - & - \\
\hline \multicolumn{5}{|c|}{$\mathrm{X}^{2}$ value } \\
\hline \multicolumn{5}{|c|}{$4.43 * *$} \\
\hline
\end{tabular}

** - Significant at 0.01 level 
Table.9 Clinical assessment for anemia of selected women residing in mining and non mining areas

\begin{tabular}{|c|c|c|c|c|c|c|}
\hline \multirow{2}{*}{$\begin{array}{l}\text { Sl } \\
\text { no }\end{array}$} & \multirow[t]{2}{*}{ Details } & \multicolumn{2}{|c|}{ Mining area } & \multicolumn{2}{|c|}{ Non mining area } & \multirow[t]{2}{*}{$\mathrm{t}$ values } \\
\hline & & $\mathbf{n}$ & $\%$ & $\mathbf{n}$ & $\%$ & \\
\hline 1 & $\begin{array}{l}\text { Non } \\
\text { interested }\end{array}$ & 41 & 58.5 & 63 & 90.0 & $4.526^{* * *}$ \\
\hline 2 & Irritation & 39 & 55.7 & 63 & 90.0 & $4.669^{* * *}$ \\
\hline 3 & Lassitude & 41 & 58.5 & 58 & 82.8 & $4.035^{* * *}$ \\
\hline 4 & Pallor skin & 41 & 58.5 & 57 & 81.4 & $3.025^{* * *}$ \\
\hline 5 & Anorexia & 10 & 14.2 & 32 & 45.7 & $4.055^{* * *}$ \\
\hline 6 & $\begin{array}{l}\text { Pale } \\
\text { conjuctiva }\end{array}$ & 10 & 14.2 & 17 & 24.2 & $1.504^{\mathrm{NS}}$ \\
\hline 7 & Koilonychia & 3 & 4.2 & 5 & 7.14 & $0.745^{\mathrm{NS}}$ \\
\hline
\end{tabular}

** - Significant at 0.01 level, NS-Non significant

Fig.1 Mean anthropometric measurements of selected women in mining and non mining areas

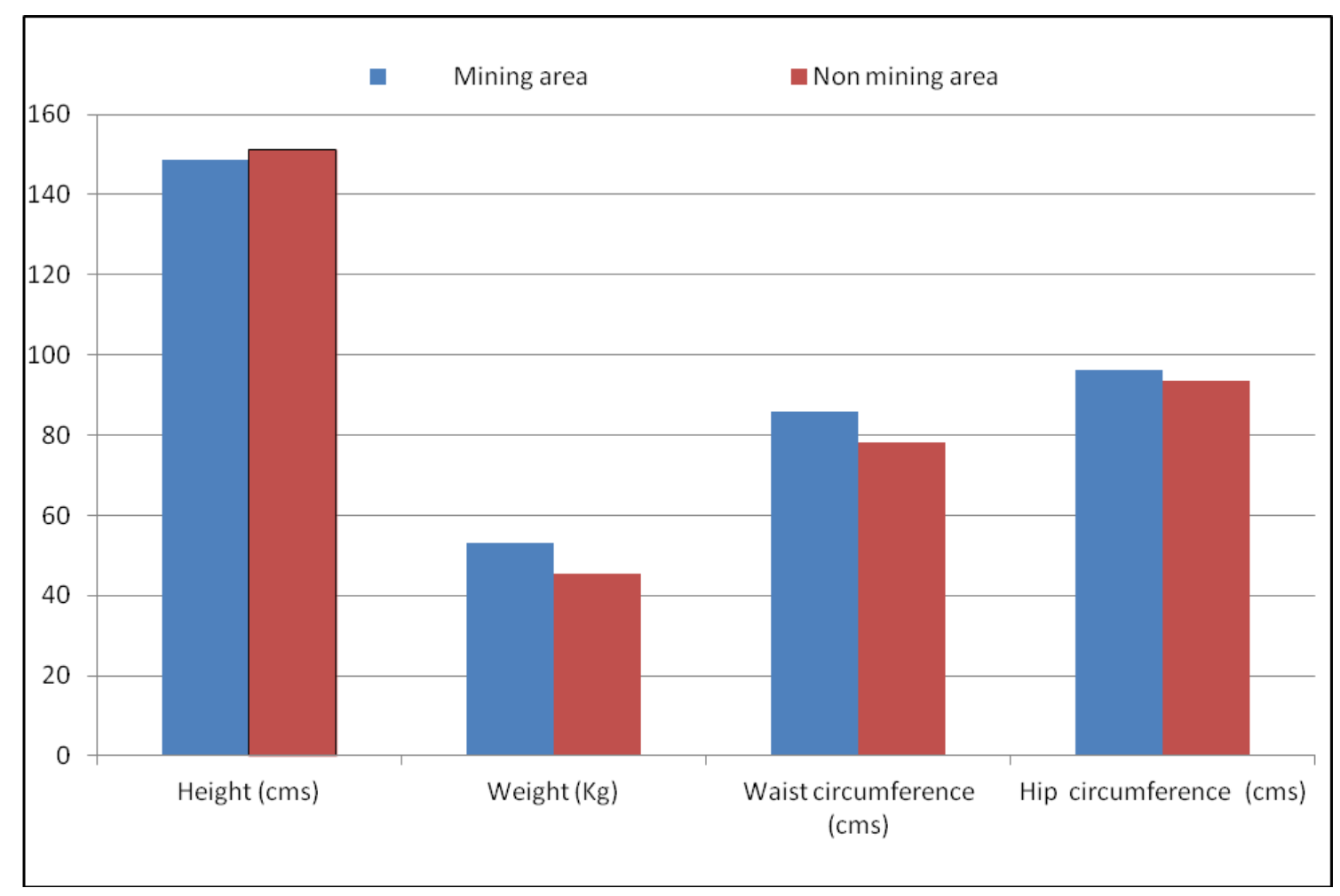


Fig.2 Per cent change in food adequacy of selected women residing in mining and non mining area

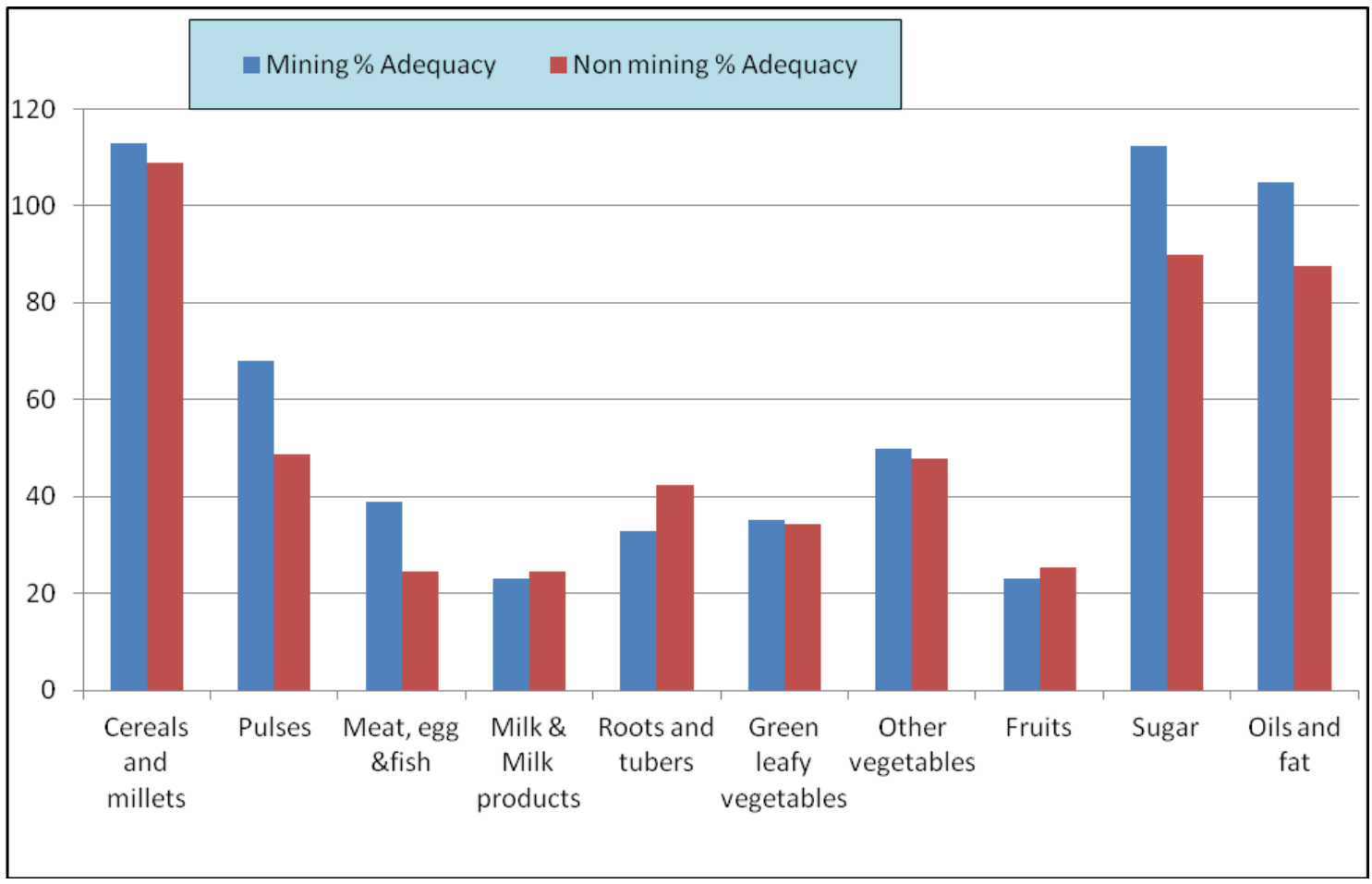

Fig.3 Mean hemoglobin level of women residing in mining and non mining area

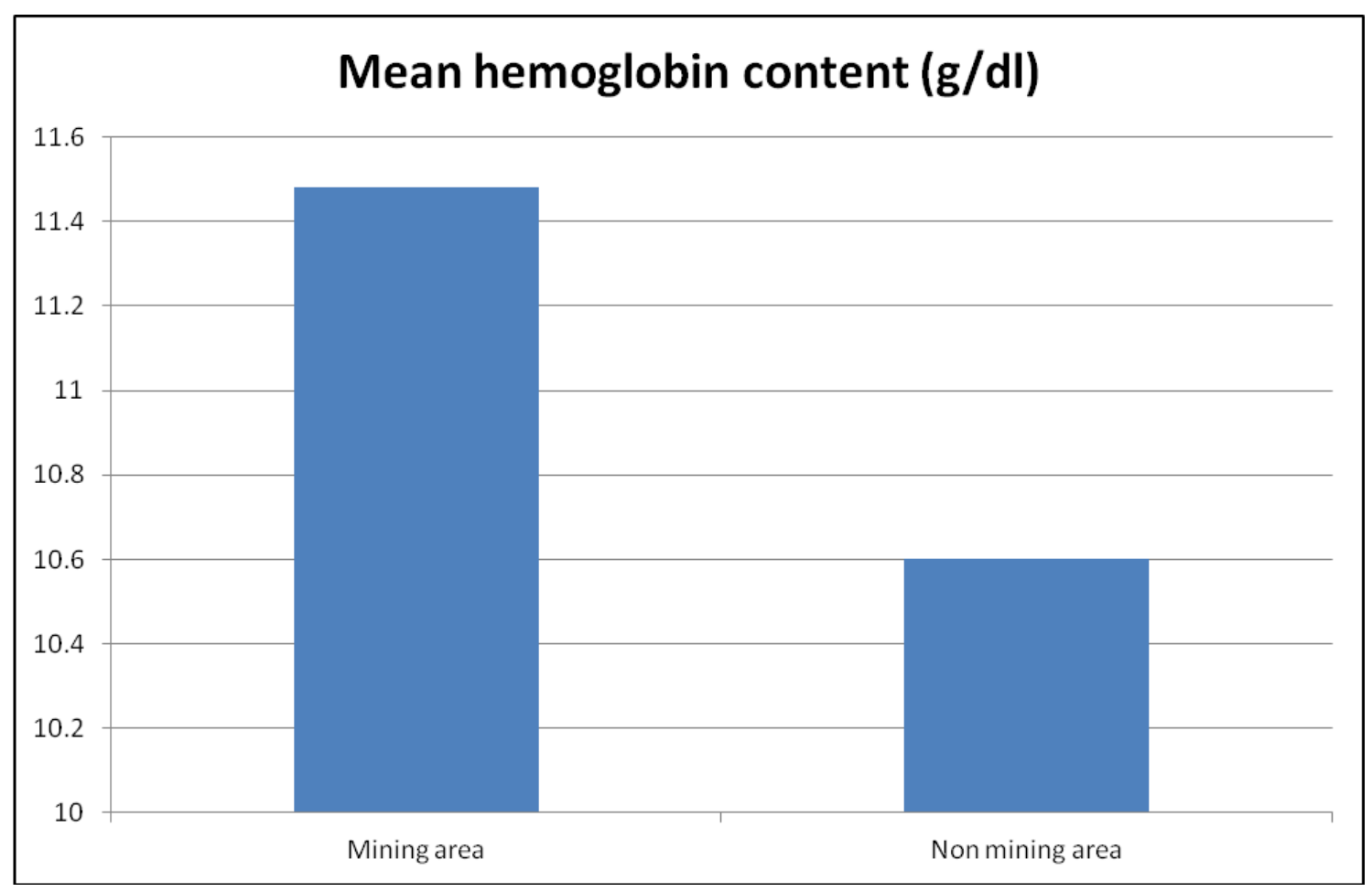


Fig.4 Clinical assessment of selected women residing in mining and non mining areas for anemia

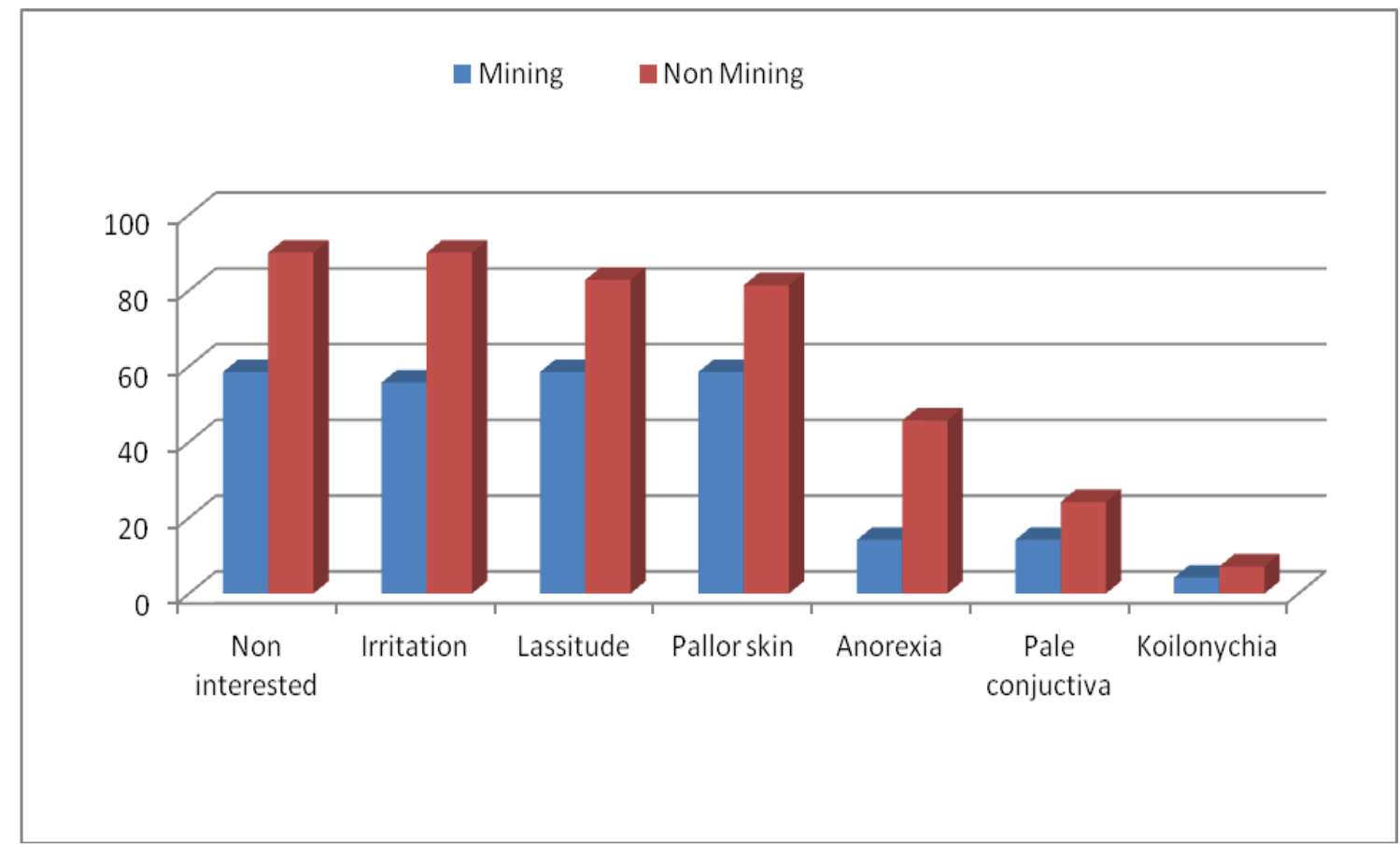

The intake of green leafy vegetables and jaggery based sweets found to be high in women residing in mining (3.04 and 2.32 respectively) area compared to non mining. The result are in confirmation with studies of Mittal, (2013) and Rao et al., (2010) and Mamata and Devi (2014).

The mean hemoglobin level of women residing in mining area was significantly higher $(11.48 \mathrm{~g} / \mathrm{dl})$ compared to women residing in non mining area $(10.06 \mathrm{~g} / \mathrm{dl})$ (Fig. 3). Both women residing in mining and non mining areas were categorized as mild anemic, but prevalence was high in women of non mining area $(55.7 \%)$ compared to mining area $(44.2 \%)$. Similar results were found in the study of Dhatrak et al., (2018) and Datt et al., (2020) where majority of the women were found to be in the range of mild to moderate anemia. Presence of clinical symptoms such as pallor skin, lassitude, lack of interest, irritation and anorexia were high (81.4, 82.8, $90.0,90.0$, and $45.7 \%$ respectively) in women residing in non mining area compared to mining area $(58.5,58.5,585$, and $57.5 \%$ respectively). Similar results were also found in the study of Sathy et al., (2017).

The study concludes that the prevalence of obesity and overweight was observed high in women residing in mining area compared to non mining area, where as prevalence of under nutrition was found to be high in women residing in non mining area. The intake of food and nutrients were significantly higher in women residing in mining area compared to non mining area. Though women are categorized as mild anemic both areas but it was found to be higher in mining area compared to non mining area.

\section{References}

Anonymous, 2003, Prevalence of Micronutrient Deficiencies, Technical Report No 22, National Institute of Nutrition Indian Council of Medical Research. Hyderabad, p. 45.

Anonymous, 2013, Impacts of mining on 
women's health, www.minesandcommunities. org, pp:14.

Anonymous, 2020, Nutritional Requirements and Recommended Dietary Allowances for Indians. Indian Council of Medical Research, Hyderabad, pp. 83.

Datrak, S. V., Nandi, S. S., Sisohodiya, P. L., Dhumne, U. L., Ingole, S. V., Gupta, S. R., 2017, Health status evaluation of mine workers and nearby population around iron ore mines in tribal district of Jharkand, India. Am. J. Prev. Med. Public. Health. 1(1): 20-26.

Datt, G., Maitra, P., Menon, N., Ray, R., Dey, S. and Chowduly, S., 2020, Impact of pollution from coal on the Anemic Status of children and women: Evidence from India, IZA institute of labour economics, Deutsche Post Foundation, pp. 1-45.

Jelliffe, D. B., 1966, The Assessment of Nutritional Status of Community, World Health Organization Monograph series No.53, Geneva, pp. 50-84.

Mamata and Devi, T. L., 2014, Prevalence of anemia and knowledge regarding anemia among reproductive age women. J. Nursing Health. Sci., 3(2): 54-60.

Mittal, M., 2013, To assess the nutritional status and morbidity pattern among non pregnant non lactating rural women of reproductive age group (18-40 years). Int. J. Sci. Res. Publication, 3(9): 1-45.

Patra, H. S., Meher, M. K. and Mohan, S. K., 2015, Socio-economic profile and quality of life of villages in and around mining area of Keonjhar district, Orissa, India. Int. Res. J. Soc. Sci., 46(6): 3139.

Rao, K. M., Balakrishna, N., Arlappa, N., Laxmaiah, A. and Brahmam, 2010, Diet and nutritional status of women in India, J. Human Ecol., 29(3): 165-170.

Sathya, P., Gandhimathi, R., Viruthasavani, K., Rodriguez, P. M., Rajeshwari, P. M., Subhatra, N. and Merlin, M. S., 2017, A study to assess the prevalence os anemia among women in a selected urban area in Coimbatore district. J. Sci. Innov. Res., 6(1): 11-15.

Vereley, H., 1976, Practical Clinical Biochemistry, Fourth Ed., Arnold Hleinemann Publications (India) Pvt. Ltd., New Delhi, pp. 45-50.

Yeboah, J.Y.B. A., 2008, Environmental and health impact of mining on surrounding communities: A case study of anglogoldashantiinobuasi. M.A. Thesis, Kwame Nkrumah Univ. Sci.Technol., Ghana.

\section{How to cite this article:}

Ullikashi, K.Y. and Kulkarni, U.N. 2021. Impact of Mining on Nutritional Status of Women of Reproductive Age Residing in Mining Affected Talukas of Ballari District, India. Int.J.Curr.Microbiol.App.Sci. 10(01): 1311-1320. doi: https://doi.org/10.20546/ijcmas.2021.1001.156 